\title{
Estudo da concordância das citações de uso e importância das espécies e famílias utilizadas como medicinais pela comunidade do bairro Ponta Grossa, Porto Alegre, RS, Brasil
}

\author{
Giovana Secretti Vendruscolo ${ }^{1,2}$ e Lilian Auler Mentz ${ }^{1}$
}

Recebido em 8/06/2004. Aceito em 26/09/2005

\begin{abstract}
RESUMO - (Estudo da concordância das citações de uso e importância das espécies e famílias utilizadas como medicinais pela comunidade do bairro Ponta Grossa, Porto Alegre, RS, Brasil). Ferramentas para quantificar dados etnobotânicos estão sendo usadas como complementares aos levantamentos sobre a utilização de plantas por populações. Neste trabalho são utilizadas técnicas para avaliar a concordância das citações de uso e a importância das espécies e famílias para as 51 pessoas entrevistadas no bairro Ponta Grossa, Porto Alegre, Rio Grande do Sul. Para tal, foram utilizados os cálculos de Valor de Uso (UV) e a porcentagem corrigida de Concordância quanto aos Usos Principais (CUPc) para as 142 espécies mencionadas no levantamento. As espécies Aloe arborescens Mill., Citrus $\times$ aurantium L., Achyrocline satureioides (Lam.) DC., Foeniculum vulgare Mill, Eugenia uniflora L., Cunila microcephala Benth., Citrus limon (L.) Osveck, Plectranthus barbatus Andrews, Cymbopogon citratus (DC.) Stapf, Psidium guajava L., Artemisia absinthium L., Ocimum basilicum L., Plantago tomentosa Lam., Rosmarinus officinalis L., Persea americana Mill., Aloysia citrodora Palau, Sambucus australis Cham. \& Schltdl., Cuphea carthagenensis (Jacq.) J.F. Macbr., Petroselinum crispum (Mill.) Nyman ex A.W. Hill, Ocimum selloi Benth. e Tanacetum vulgare L., nesta ordem de Valor de Uso, foram consideradas como as mais importantes para a população estudada. As famílias mais importantes foram Asphodelaceae, Caprifoliaceae, Rutaceae e Lythraceae. Para o cálculo da porcentagem a corrigida de Concordância quanto aos Usos Principais $\left(\mathrm{CUP}_{\mathrm{c}}\right)$ foram consideradas como espécies principais as que apresentaram valores acima de 24\%: Eugenia uniflora, Achyrocline satureioides, Psidium guajava, Cunila microcephala, Plectranthus barbatus, Citrus $\times$ aurantium, Citrus limon, Cymbopogon citratus, Punica granatum L., Sechium edule (Jacq.) Sw., Sphagneticola trilobata (L.) Pruski, Aloysia citrodora, Foeniculum vulgare, Plectranthus neochilus Schltr., Artemisia absinthium, Lippia alba (Mill.) N.E. Br., Mikania laevigata Sch. Bip ex Baker, Aloe arborescens e Petroselinum crispum.
\end{abstract}

Palavras-chave: plantas medicinais, Valor de Uso, Concordância de Uso

\begin{abstract}
Study of use citations agreement and importance of medicinal used species and families to the community of Ponta Grossa neighborhood, Porto Alegre, Rio Grande do Sul State, Brazil). Tools are being used as complement to the study of plant usage by communities to quantify ethnobotanical data. We utilized techniques to evaluate informants use citations agreement and the importance of species and families to the 51 interviewed persons of the community of Ponta Grossa neighborhood, Porto Alegre, Rio Grande do Sul, Brazil. Then we have utilized the Use Value (UV) method and the corrected percentage of Agreement related to the Main Uses (cAMU) for the 142 species mentioned in this work. In order of Use Value, the Aloe arborescens Mill., Citrus $\times$ aurantium L., Achyrocline satureioides (Lam.) DC., Foeniculum vulgare Mill, Eugenia uniflora L., Cunila microcephala Benth., Citrus limon (L.) Osveck, Plectranthus barbatus Andrews, Cymbopogon citratus (DC.) Stapf, Psidium guajava L., Artemisia absinthium L., Ocimum basilicum L., Plantago tomentosa Lam., Rosmarinus officinalis L., Persea americana Mill., Aloysia citrodora Palau, Sambucus australis Cham. \& Schltdl., Cuphea carthagenensis (Jacq.) J.F. Macbr., Petroselinum crispum (Mill.) Nyman ex A.W. Hill, Ocimum selloi Benth. and Tanacetum vulgare L. species were considered the most important to the studied community. The most important families were Asphodelaceae, Caprifoliaceae, Rutaceae and Lythraceae. We have considered as main species those that presented at least $24 \%$ of corrected percentage of Agreement related to the Main Uses (cAMU). They are: Eugenia uniflora, Achyrocline satureioides, Psidium guajava, Cunila microcephala, Plectranthus barbatus, Citrus $\times$ aurantium, Citrus limon, Cymbopogon citratus, Punica granatum L., Sechium edule (Jacq.) Sw., Sphagneticola trilobata (L.) Pruski, Aloysia citrodora, Foeniculum vulgare, Plectranthus neochilus Schltr., Artemisia absinthium, Lippia alba (Mill.) N.E. Br., Mikania laevigata Sch. Bip ex Baker, Aloe arborescens and Petroselinum crispum.
\end{abstract}

Key words: medicinal plants, Use Value, Use Agreement

\section{Introdução}

Nos últimos anos, técnicas quantitativas têm sido aplicadas como informações complementares aos levantamentos etnobotânicos (Alexiades 1996). Phillips (1996) define a etnobotânica quantitativa como a utilização de técnicas estatísticas para analisar dados de uso das plantas. A etnobotânica

\footnotetext{
1 Departamento de Botânica, Instituto de Biociências, Universidade Federal do Rio Grande do Sul (UFRGS), Bairro Agronomia, Av. Bento Gonçalves 9500, Bloco IV, CEP 91509-900, Porto Alegre, RS, Brasil

2 Autor para correspondência: gvendruscolo@yahoo.com.br
} 
quantitativa pode ser usada com vários objetivos, tais como avaliar a importância das plantas para um determinado grupo étnico, comparar usos e/ou comunidades vegetais entre diferentes populações, comparar a importância de diferentes tipos de vegetação para uma comunidade, estabelecer e comparar a importância relativa de espécies e famílias de plantas medicinais, entre outras (Phillips \& Gentry 1993).

Dados quantitativos podem ser usados como justificativa para a conservação das espécies vegetais e do conhecimento popular, principalmente, por fornecerem informações sobre as espécies e/ou famílias mais utilizadas para diversos fins. Através da técnica de Valor de Uso - UV - (Phillips \& Gentry 1993), pode-se inferir quais são as espécies e/ou famílias mais importantes para uma população. $\mathrm{O}$ critério para avaliação desta importância é o "uso" de uma espécie pelos informantes. Portanto, quanto mais usos forem mencionados para uma espécie, maior importância ela terá para a comunidade. Esta técnica também pode ser usada para selecionar espécies para cultivos em hortos comunitários e/ou confecção de manuais com informações científicas sobre plantas medicinais, propiciando uma contribuição do pesquisador à população estudada. Outra técnica importante está relacionada com a confiabilidade das indicações de usos medicinais mencionadas pelos informantes para as espécies. Segundo Alexiades (1996), os usos medicinais mais confiáveis são aqueles já utilizados pelos informantes, parentes ou conhecidos do mesmo. Outro critério para selecionar o uso mais importante de uma espécie e as espécies que possuem maior potencial para um determinado uso é o Índice de Fidelidade, criado por Friedman et al. (1986). O Índice de Fidelidade pode ser uma solução eficiente para a seleção de plantas a serem estudadas farmacologicamente.

O presente trabalho tem o objetivo de utilizar métodos quantitativos para detectar as espécies e as famílias mais importantes para a população do bairro Ponta Grossa e aquelas que a população considera com maior potencial de cura para um determinado uso. As espécies mais importantes para a comunidade foram utilizadas para a realização de um trabalho, que relacionou as informações encontradas na literatura científica sobre composição química e atividades farmacológicas destas plantas com as indicações de usos medicinais mencionados pela população (Vendruscolo et al. 2005), servindo de base para a confecção futura de um manual dirigido aos informantes.

\section{Material e métodos}

Foi realizado um levantamento das plantas utilizadas como medicinais por moradores do bairro Ponta Grossa, Porto Alegre, Rio Grande do Sul e pelos Agentes Comunitários de Saúde que prestam assistência junto ao Posto de Saúde da Família do bairro. As entrevistas foram feitas nas oito regiões onde atuam os agentes de saúde. Cada agente de saúde faz visitas aos moradores de uma das regiões prédefinidas pelos responsáveis pelo Posto de Saúde. Cada região é constituída por aproximadamente 200 residências, todas cadastradas no Posto. Para cada região foram realizadas seis ou sete entrevistas (incluindo o agente correspondente), totalizando 51 informantes (G.S. Vendruscolo, dados não publicados). As exsicatas das plantas coletadas durante as entrevistas foram incluídas no herbário ICN da Universidade Federal do Rio Grande do Sul e os números de coleta estão mencionados em G.S. Vendruscolo (dados não publicados).

Para cada espécie foi calculado o Valor de Uso $\left(U_{\mathrm{s}}\right)$ conforme referido por Phillips \& Gentry (1993). Para o cálculo do Valor de Uso de uma espécie para um informante $\left(U V_{i s}\right)$ foi utilizada a fórmula $U V_{\text {is }}$ $=\Sigma U_{\text {is }} / n_{\text {is }}$, onde $U_{\text {is }}$ corresponde ao número de usos mencionados pelo informante para a espécie e $n_{\text {is }}$ ao número de entrevistas feitas com o informante. Para este trabalho, $\mathrm{n}_{\mathrm{is}}$ é sempre 1 (um) para todas as espécies, pois somente uma entrevista por informante foi realizada. Portanto, o valor de $\mathrm{UV}_{\text {is }}$ será igual ao de $\mathrm{U}_{\text {is }}$.

Para o cálculo do Valor de Uso de cada espécie $\left(U V_{s}\right)$ foi utilizada a fórmula $U_{\mathrm{s}}=\Sigma \mathrm{UV}_{\mathrm{is}} / \mathrm{n}$, onde $U_{\text {is }}$ equivale ao valor de uso de uma espécie para um informante e $n$ é o número total de informantes entrevistados. $\mathrm{O}$ valor de $\mathrm{n}$ corresponde ao valor de $\mathrm{n}_{\mathrm{s}}$ referido por Phillips \& Gentry (1993), assumindo-se a posição de que todas as espécies poderiam ser citadas por qualquer informante.

Para o cálculo do Valor de Uso da família botânica (FUV) foi utilizada a alternativa proposta por Phillips \& Gentry (1993), em que são estimadas as médias dos Valores de Uso das espécies pertencentes a uma família, ou seja, FUV = " $U V_{\mathrm{s}} / \mathrm{n}_{\mathrm{f}}$ (onde $\mathrm{n}_{\mathrm{f}}$ é o número de espécies registradas para a família).

Para avaliar o Índice de Fidelidade dos usos mencionados para cada espécie foi utilizada a metodologia proposta por Friedman et al. (1986) e modificada por Amorozo \& Gély (1988). Para estimar o Índice de Fidelidade, utilizou-se o cálculo de 
porcentagem de Concordância quanto aos Usos Principais - CUP - (mais citados) para a espécie, usando-se o número de informantes que citaram o uso principal vezes 100 , dividido pelo número de informantes que citaram a espécie. Devido às diferenças no número de informantes que citaram usos para cada espécie, é necessária a utilização de um Fator de Correção (FC). O Fator de Correção é igual ao número de informantes que citaram usos para a espécie, dividido pelo número de informantes que citaram a espécie principal, ou seja, com maior número de usos referidos. Portanto, para calcular a porcentagem de Concordância corrigida quanto aos Usos Principais para cada uma das espécies (CUPc) é utilizada a multiplicação de CUP e FC.

As espécies foram listadas em uma tabela onde constam em ordem alfabética, seguidas da família e dos nomes populares, constando entre parênteses os números correspondentes às oito regiões em que o bairro é subdividido. Os usos populares estão representados de forma literal, ou seja, como foram referidos pelos informantes e em ordem alfabética por categoria de uso, também seguidos da região em que foram mencionados. Foram utilizadas as categorias medicinal (Med), aromatizante (Aro), místico (Mis), alimentação (Ali) e tempero (Tem).

\section{Resultados e discussão}

No levantamento realizado com moradores e Agentes Comunitários de Saúde, que prestam assistência junto ao Posto de Saúde da Família, do bairro Ponta Grossa, Porto Alegre, Rio Grande do Sul (G.S. Vendruscolo, dados não publicados), foram identificadas 142 espécies, pertencentes a 59 famílias (Tab. 1).

Segundo o critério do cálculo de Valor de Uso, o número de usos mencionados para uma espécie estabelece a importância dela para a comunidade estudada. Devido a isto, quanto maior o número de usos, independente da categoria, mencionados para a espécie, maior será a importância da mesma para a comunidade. As 21 espécies consideradas mais importantes para a população estudada, em ordem de Valor de Uso, são: Aloe arborescens (originária da África), Citrus $\times$ aurantium (originária da Ásia), Achyrocline satureioides (originária da Ámerica do Sul), Foeniculum vulgare (originária da Europa), Eugenia uniflora (originária da América do Sul), Cunila microcephala (originária da América do Sul), Citrus limon (originária da Ásia), Plectranthus barbatus (originária da África), Cymbopogon citratus (originária da Ásia), Psidium guajava (originária da América tropical), Artemisia absinthium (originária da Eurásia), Ocimum basilicum (originária da Eurásia), Plantago tomentosa (originária da América do Sul), Rosmarinus officinalis (originária da Europa), Persea americana (originária da América tropical), Aloysia citrodora (originária da América do Sul), Sambucus australis (originária da América do Sul), Cuphea carthagenensis (originária da América do Sul), Petroselinum crispum (originária da Europa), Ocimum selloi (originária da América do Sul) e Tanacetum vulgare (originária da Europa).

Das 21 espécies citadas, 10 (48\%) são nativas nas Américas e 11 delas têm origem em outros continentes. Das espécies nativas nas Américas, oito são nativas no Estado do Rio Grande do Sul, estando estas bem representadas entre as espécies mais importantes para a população do bairro. Com exceção de Achyrocline satureioides ("marcela") e Cuphea carthagenensis ("sete-sangria"), as outras espécies com maior índice de importância para a comunidade são cultivadas nos pátios e/ou jardins e ainda adquiridas com vizinhos e amigos que as cultivam. A marcela é coletada nos campos ou em beiras de estrada tradicionalmente na época religiosa da Semana Santa (uma semana antes da Páscoa), e suas flores são postas para secar e posteriormente guardadas para utilização. Cuphea carthagenensis ocorre nos campos e beiras de estrada, não havendo a necessidade do plantio.

Aloe arborescens, Achyrocline satureioides, Citrus limon, Citrus $\times$ aurantium, Cunila microcephala, Eugenia uniflora, Foeniculum vulgare e Plectranthus barbatus foram citadas por informantes pertencentes a todas as oito regiões em que o bairro é dividido pelo Posto de Saúde, possuindo cada uma delas, mais de 30 citações de uso. Este dado demonstra que o conhecimento sobre a utilização destas espécies é igualmente distribuído no bairro. As famílias consideradas mais importantes para a população do bairro Ponta Grossa, conforme o Valor de Uso da Família (FUV), são Asphodelaceae, Caprifoliaceae, Rutaceae, Lythraceae, Apiaceae, Piperaceae, Plantaginaceae, Lauraceae, Lamiaceae e Punicaceae.

O cálculo do Valor de Importância de uma Família é feito através da média dos Valores de Uso das espécies encontradas para esta. Desta forma, famílias com grande número de espécies citadas não necessariamente serão consideradas as mais importantes para uma comunidade (Phillips \& Gentry 


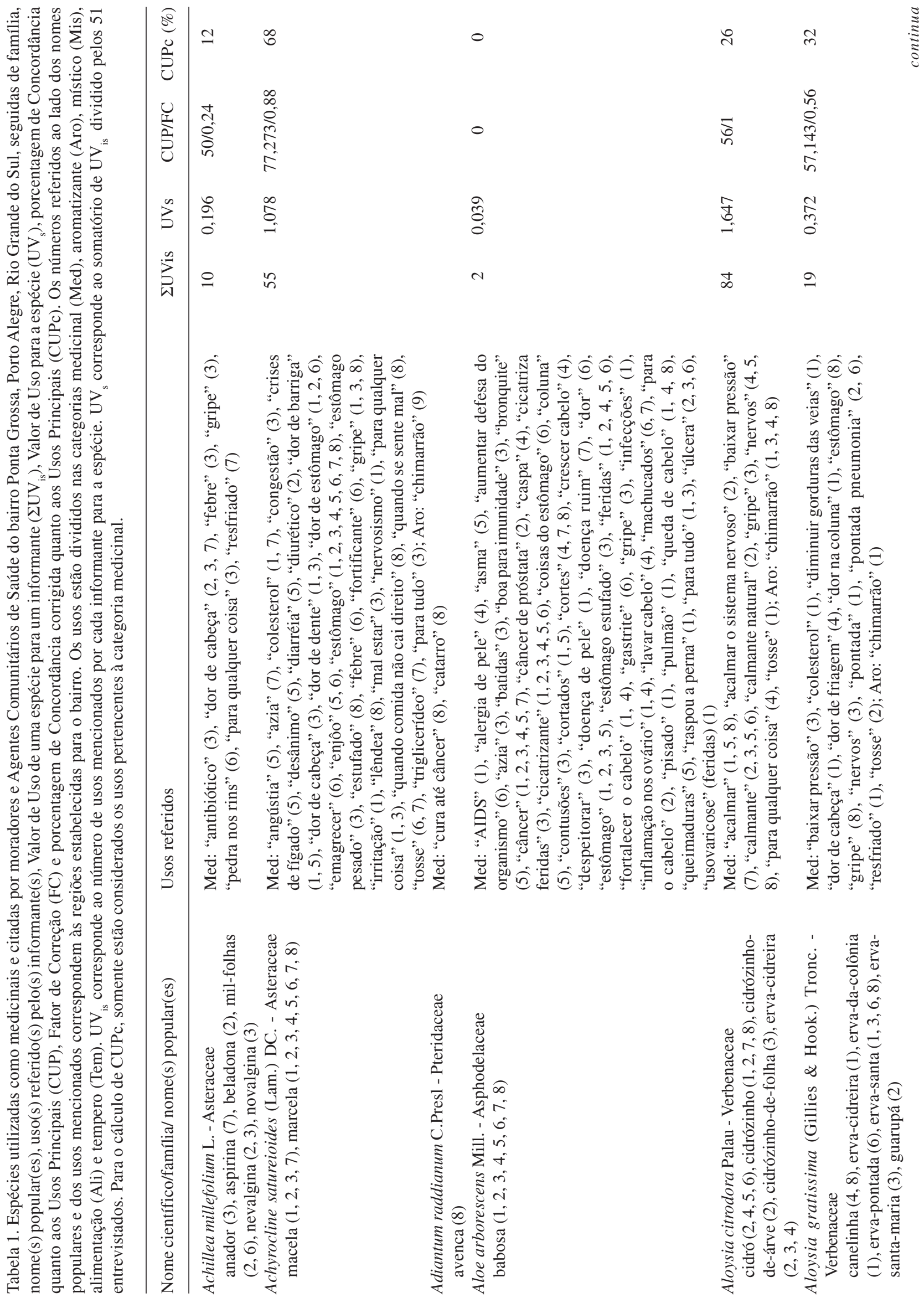




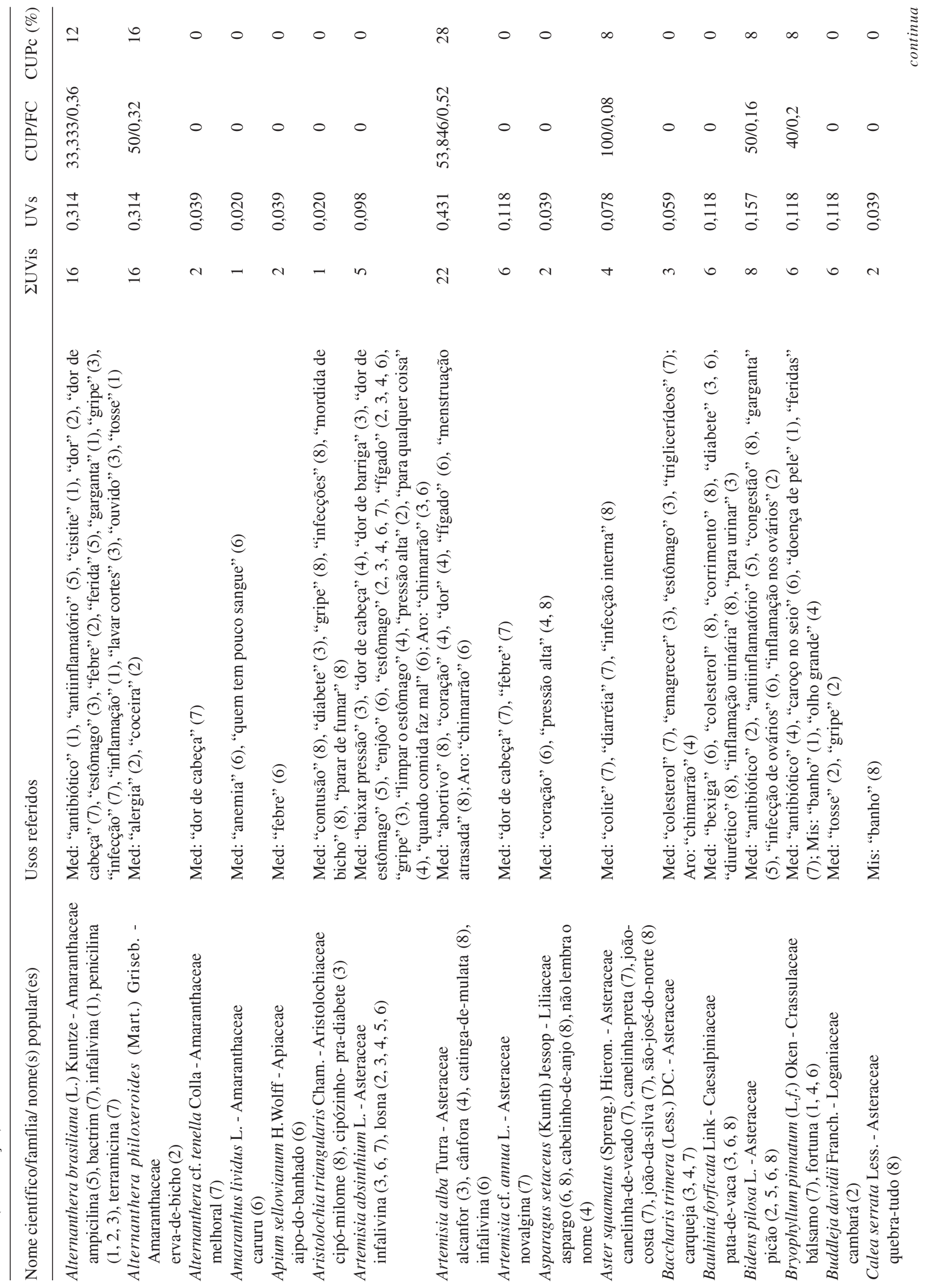


Vendruscolo \& Mentz: Estudo da concordância das citações de uso e importância das espécies e famílias...

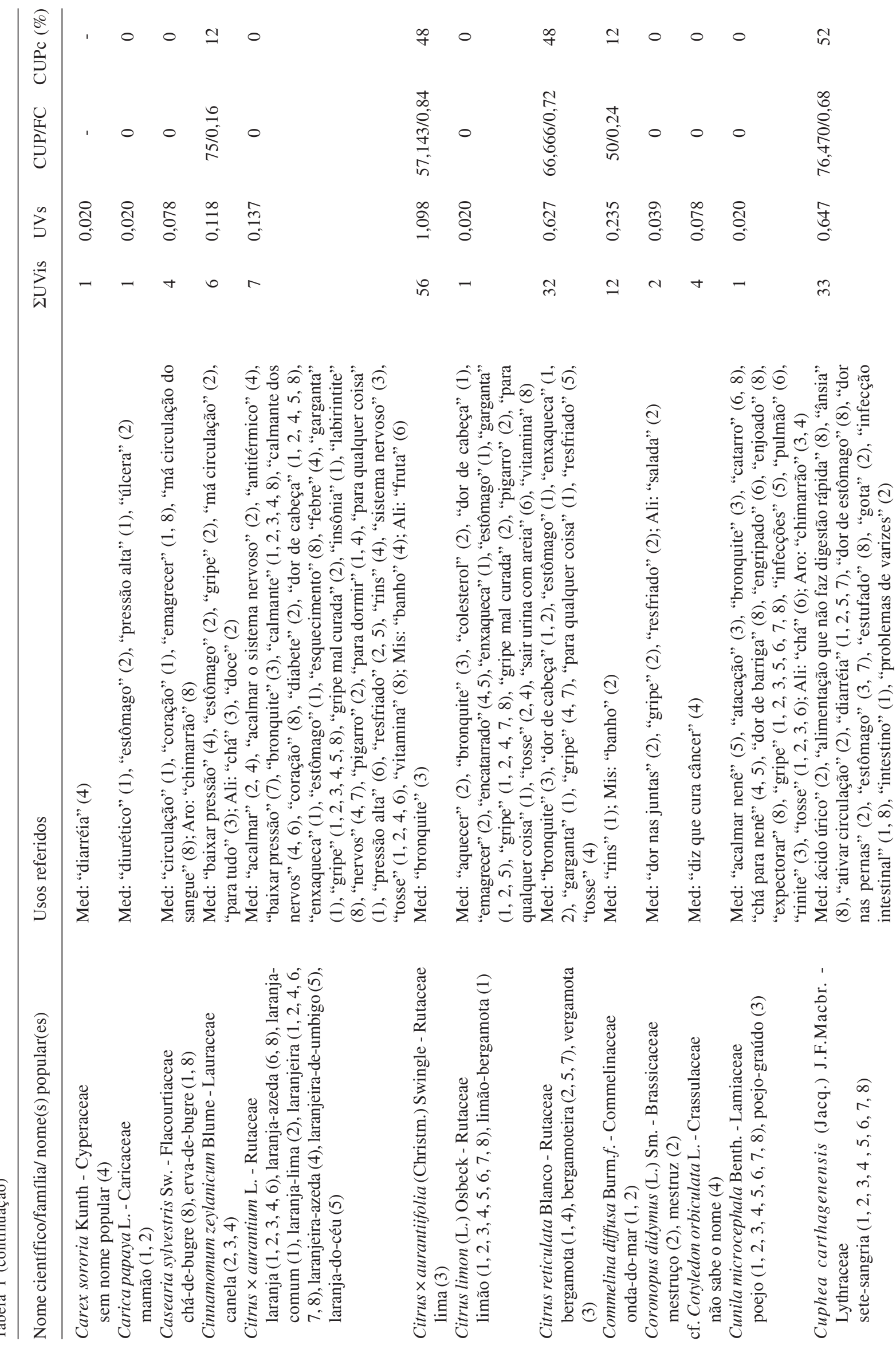




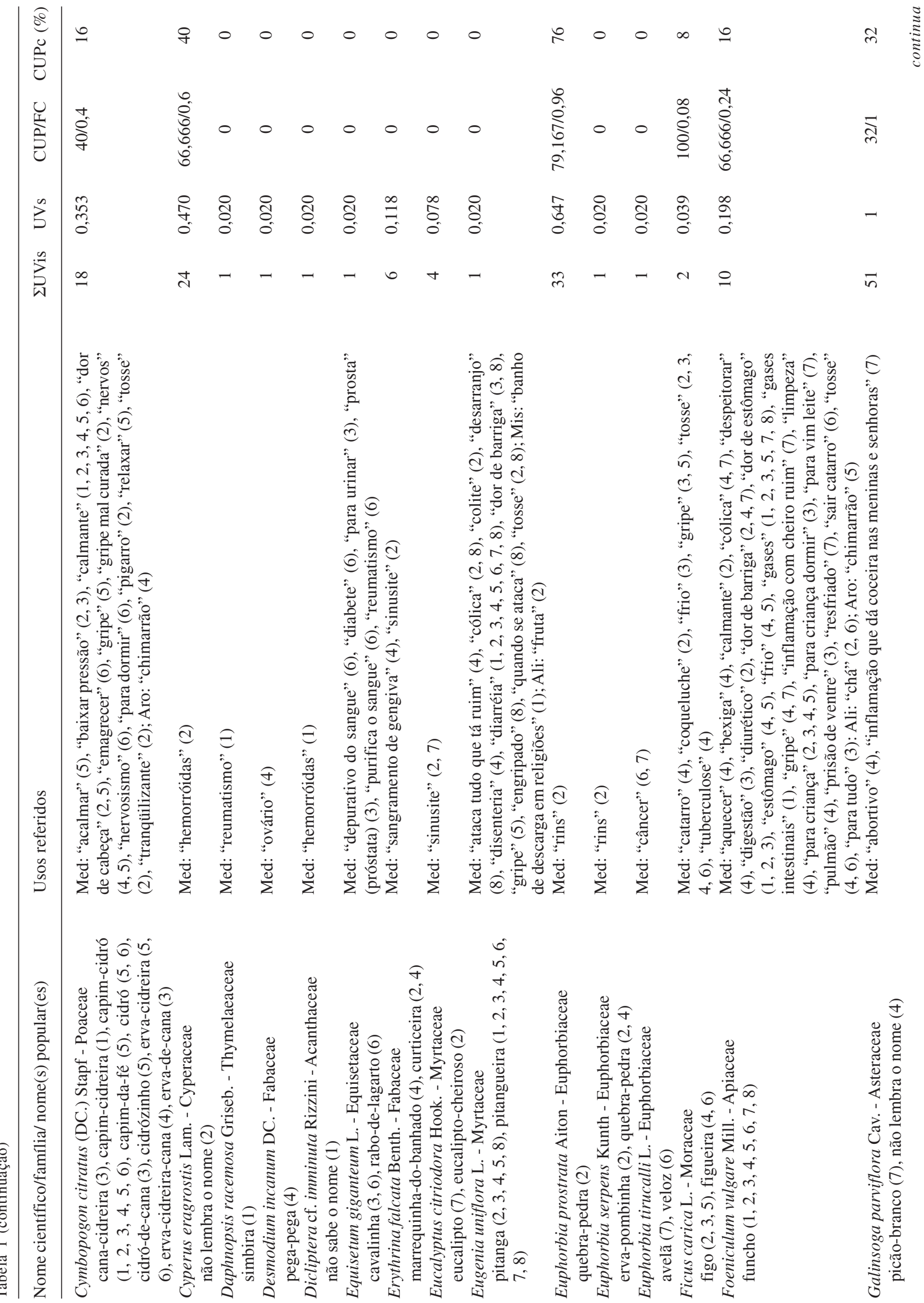




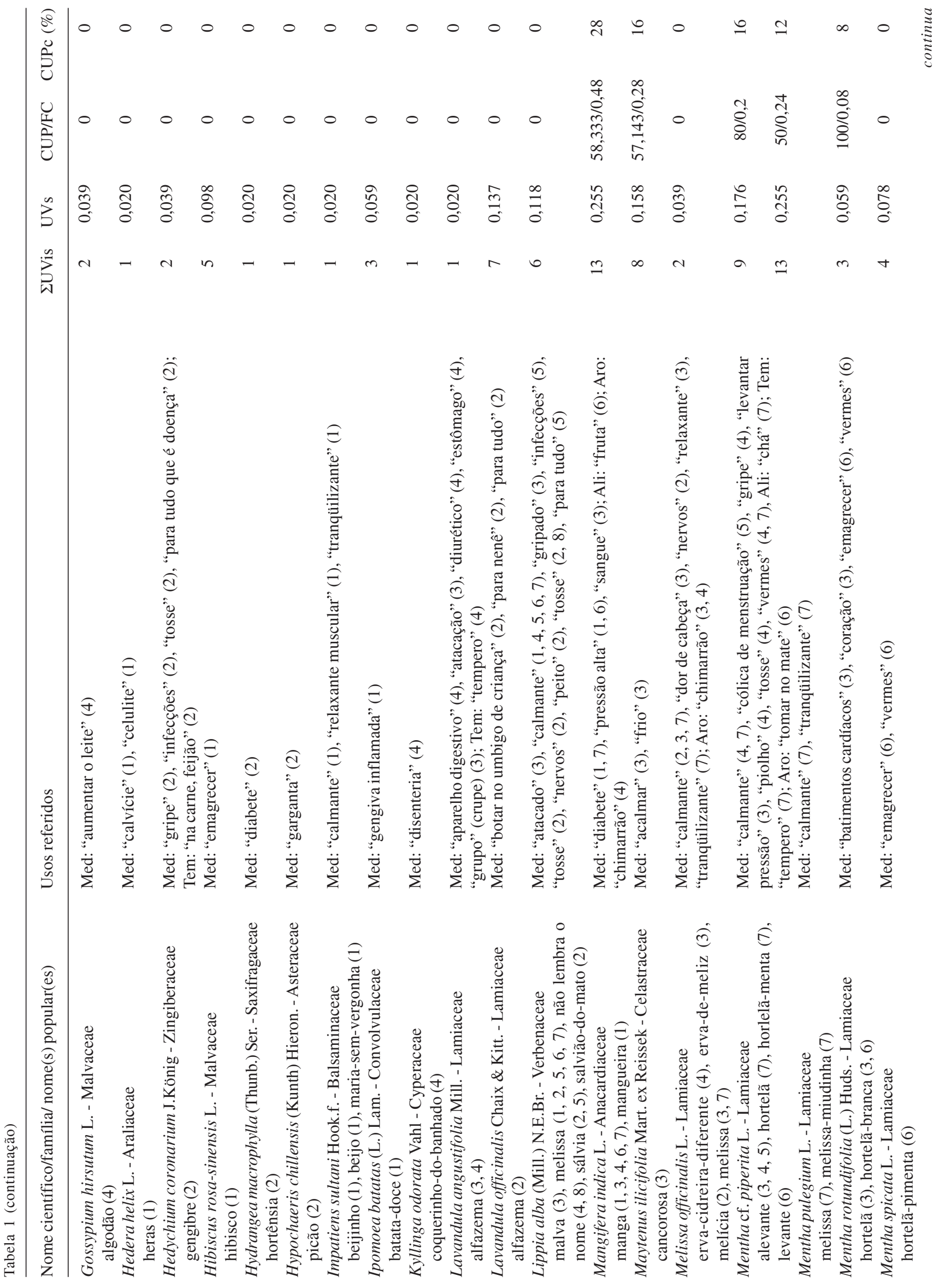




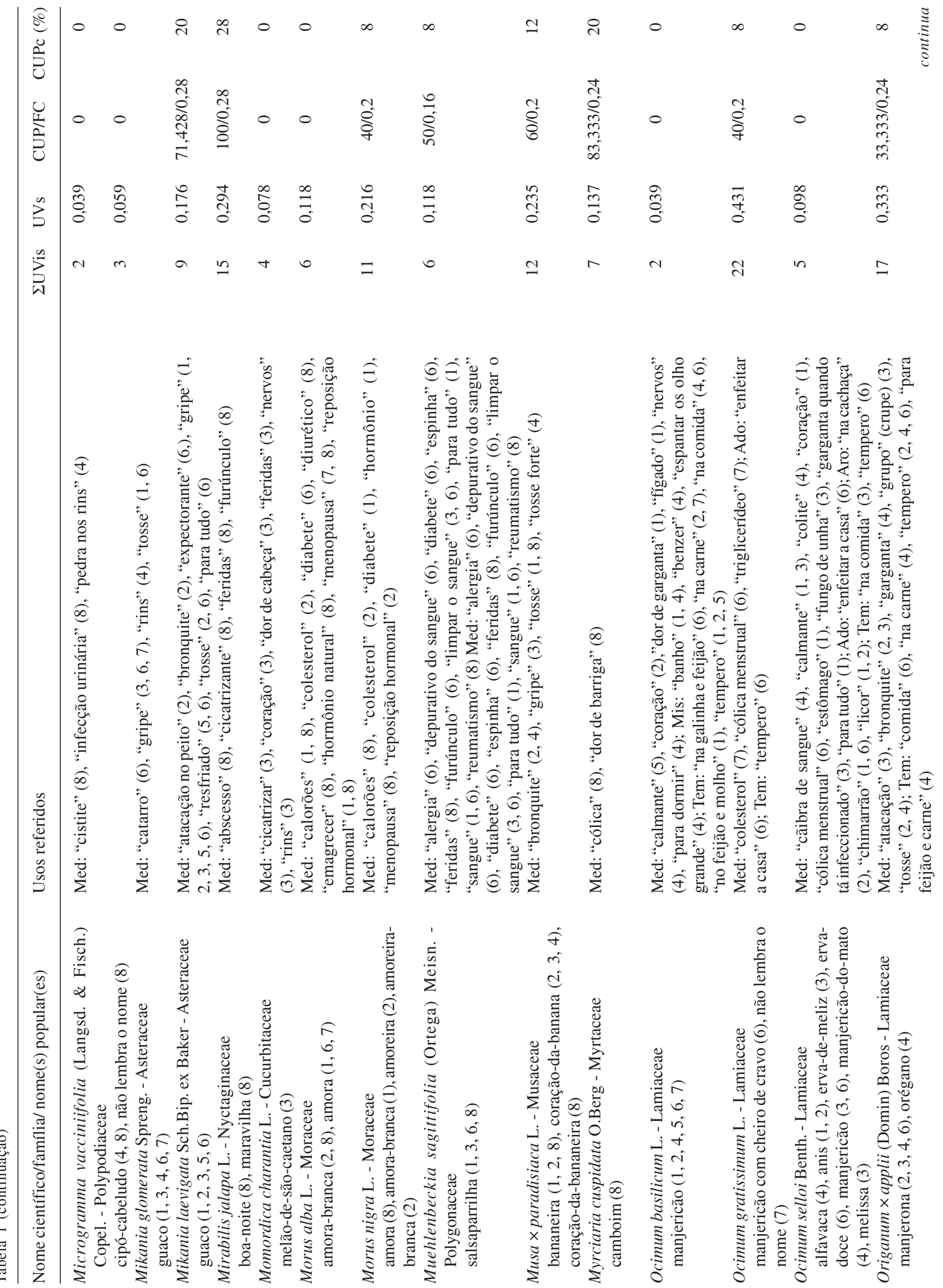




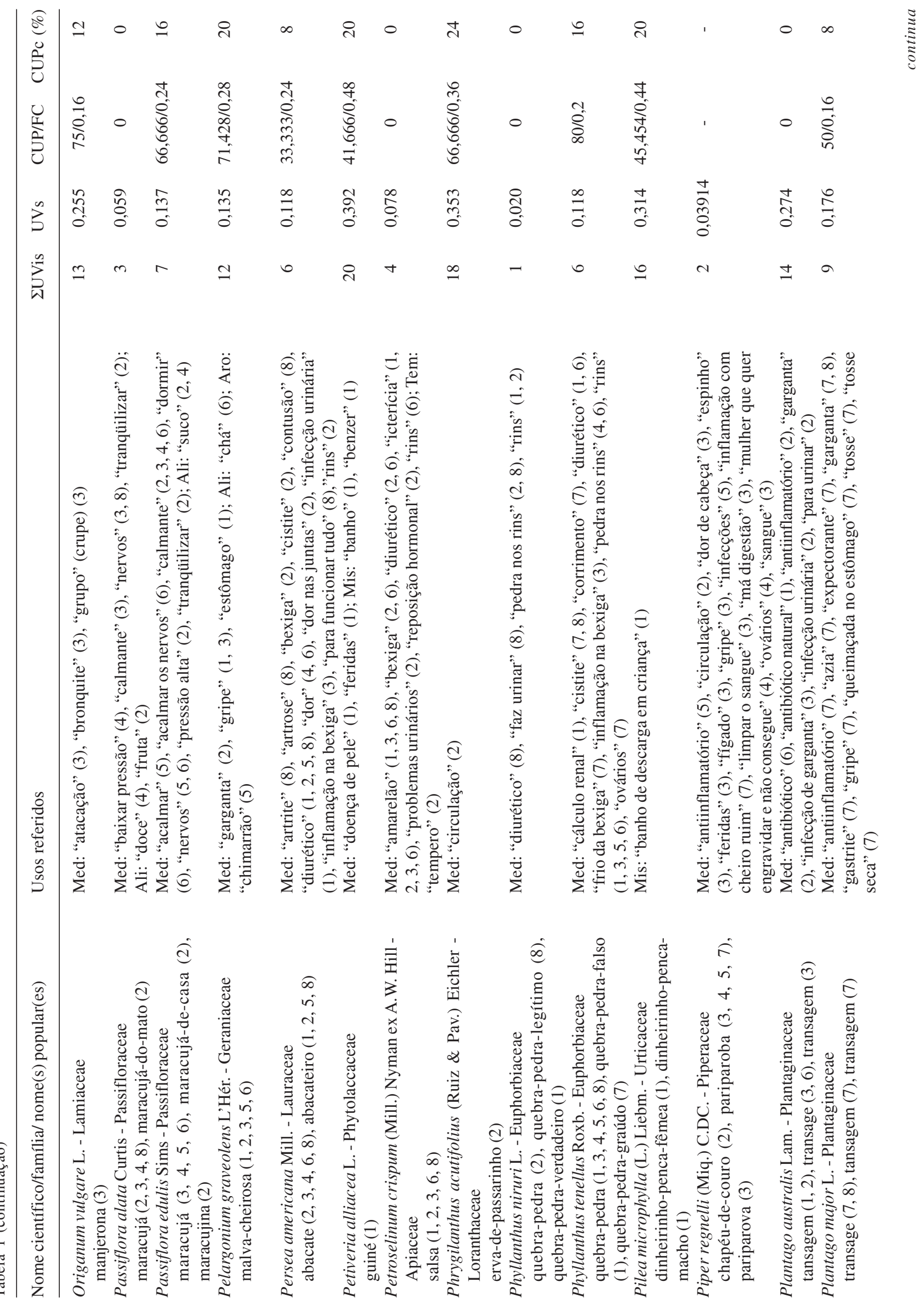



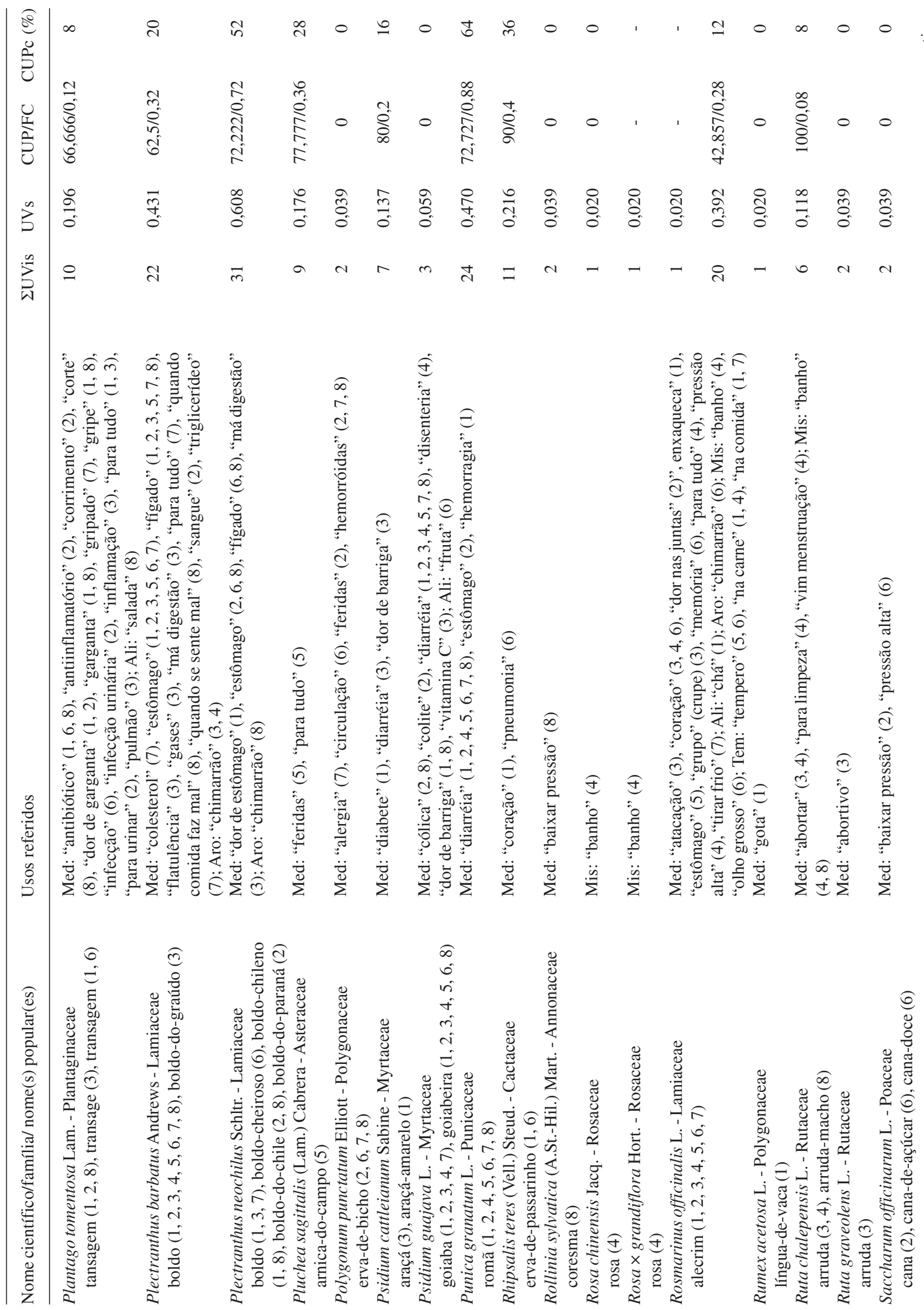


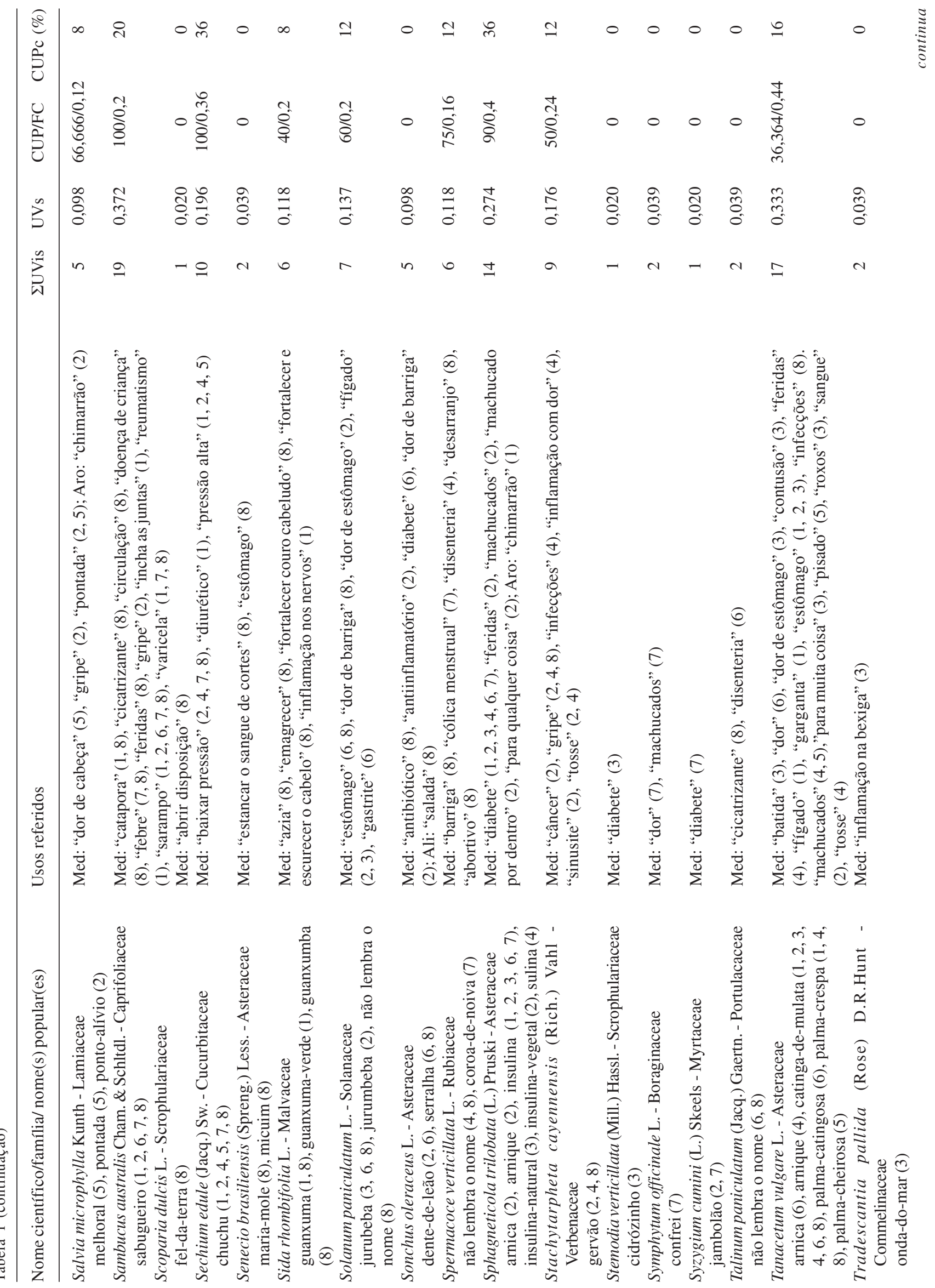




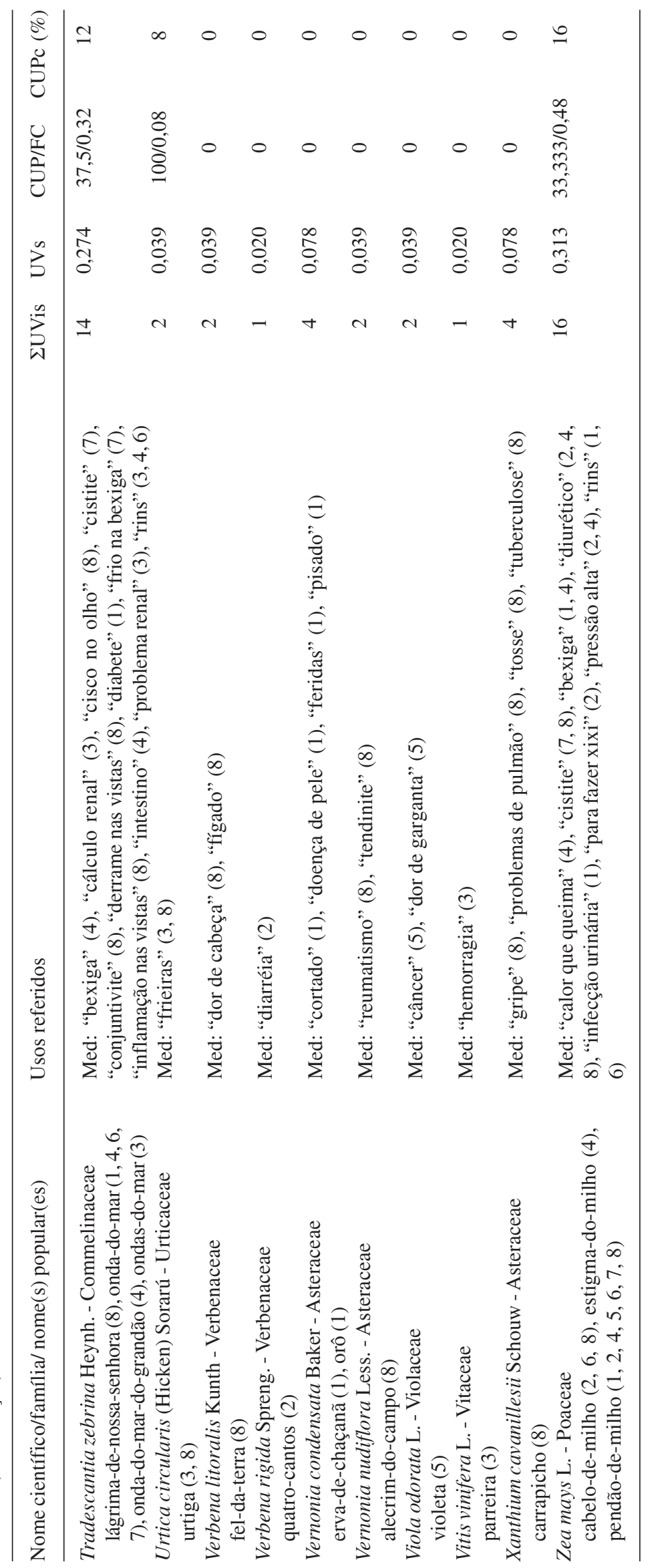


1993). Existe uma relação direta entre as espécies e as famílias consideradas como mais importantes para uma população. Para a família Asphodelaceae somente foi citada Aloe arborescens (babosa). Porém, esta espécie possui o maior índice de Valor de Uso entre todas as espécies, sendo portanto Asphodelaceae a família considerada mais importante para a comunidade. Este fato também ocorre com Caprifoliaceae e Lythraceae, famílias em que foram mencionadas somente Sambucus australis e Cuphea carthagenensis, respectivamente. As outras famílias, exceto Piperaceae e Punicaceae, possuem um número maior de espécies citadas, porém uma ou mais delas estão representadas entre as 21 espécies mais importantes. Já a família Asteraceae possui o maior número de espécies citadas (21), sendo que somente 3 delas estão incluídas entre as $21 \mathrm{com}$ maior Valor de Uso. Portanto, ela está classificada como a $13^{\text {a }}$ família mais importante para a população do bairro.

As espécies com maior porcentagem de Concordância corrigida quanto aos Usos Principais para cada uma das espécies (CUPc) estão representadas na tabela 2 . O valor 0 (zero) da porcentagem de CUPc referido para algumas espécies, significa que a mesma somente foi mencionada por um dos informantes ou citada por mais de um informante sem haver coincidências entre seus usos.
No primeiro caso, não se pode estimar a concordância entre os usos e no segundo, não houve esta concordância.

Quanto maior o valor da porcentagem de Concordância Corrigida quanto aos Usos Principais para cada uma das espécies (CUPc), maior é o número de informantes que citou o uso principal para a espécie, portanto com maior concordância da população na indicação deste uso. Como o $\mathrm{CUP}_{\mathrm{c}}$ é um índice de fidelidade quanto ao uso principal referido pelos informantes para a espécie, ele pode indicar quais as espécies mais promissoras para a realização de estudos farmacológicos relativos aos usos indicados pela população.

Os valores percentuais de Concordância corrigida quanto aos Usos Principais para cada uma das espécies (CUPc) também foram utilizados em outros levantamentos etnobotânicos realizados no estado. As espécies que também foram encontradas nestes trabalhos com o índice de CUPc maior que $40 \%$ são Achyrocline satureioides (R.R. Kubo, dados não publicados; R.M. Possamai, dados não publicados), Citrus $\times$ aurantium (S.M. Marodin, dados não publicados - como Citrus sinensis (L.) Osbeck), Cunila microcephala (S.M. Marodin, dados não publicados; Possamai 2000), Eugenia uniflora (R.M. Possamai, dados não publicados) e Psidium guajava

Tabela 2. Espécies com valores percentuais de Concordância Corrigida quanto aos Usos Principais (CUPc) maiores que 24\%, em ordem decrescente, encontradas no levantamento realizado com os moradores do bairro Ponta Grossa, Porto Alegre, Rio Grande do Sul. Os usos referidos na tabela são os usos considerados principais, ou seja, citados por um maior número de informantes. A parte da planta referida corresponde a utilizada conforme o uso principal. Os usos estão citados de forma literal.

\begin{tabular}{|c|c|c|c|}
\hline Espécies & $\operatorname{CUP}_{\mathrm{c}}(\%)$ & Parte utilizada & Usos principais \\
\hline Eugenia uniflora & 76 & folhas & contra "diarréia", "disenteria" e "desarranjo" \\
\hline Achyrocline satureioides & 68 & inflorescências & para problemas de "estômago" \\
\hline Psidium guajava & 64 & folhas & contra "diarréia" e "disenteria" \\
\hline Cunila microcephala & 52 & partes aéreas & contra "gripe" \\
\hline Plectranthus barbatus & 52 & folhas & para problemas de "estômago" \\
\hline Citrus $\times$ aurantium & 48 & folhas & como "calmante" e para "nervos" \\
\hline Citrus limon & 48 & frutos & contra "gripe" \\
\hline Cymbopogon citratus & 40 & folhas & como "calmante", "tranqüilizante" e para nervos" \\
\hline Punica granatum & 36 & casca do fruto & contra "diarréia", \\
\hline Sechium edule & 36 & folhas, frutos & para "baixar pressão" e contra "pressão alta" \\
\hline Sphagneticola trilobata & 36 & partes aéreas & contra "diabete" \\
\hline Aloysia citrodora & 32 & folhas & como "calmante" e para "nervos" \\
\hline Foeniculum vulgare & 32 & partes aéreas & contra "gases" ou "gases intestinais" \\
\hline Plectranthus neochilus & 28 & folhas & para problemas do "estômago" \\
\hline Artemisia absinthium & 28 & partes aéreas & para problemas do "estômago" \\
\hline Lippia alba & 28 & folhas & como "calmante" e para "nervos" \\
\hline Mikania laevigata & 28 & folhas & contra "gripe" \\
\hline Aloe arborescens & 26 & folhas & contra "feridas", "machucados" e como cicatrizante \\
\hline Petroselinum crispum & 24 & raízes & contra "amarelão" ou "icterícia" \\
\hline
\end{tabular}


(R.M. Possamai, dados não publicados).

Neste trabalho, Eugenia uniflora (76\%), Achyrocline satureioides (68\%) e Psidium guajava (64\%) apresentaram maiores valores de CUPc. Os usos mais citados para estas espécies foram contra "diarréia", "disenteria", "desarranjo" e para problemas relacionados ao "estômago". Para 19 espécies com valor de CUPc maior que $24 \%$, o uso principal de quatro delas está relacionado com problemas do "estômago" e como "calmante", três delas são usadas contra "diarréia" e "gripe" e uma delas para "baixar pressão", contra "diabete", "gases", "feridas" e "amarelão" ou "icterícia".

Para Calea serrata, Pilea microphyla, Rosa chinensis e Rosa $\times$ grandiflora o índice de Concordância quanto aos Usos Principais corrigido para cada uma das espécies (CUPc) não pode ser calculado, porque para elas não foram mencionados usos medicinais. Os usos referidos pela comunidade do bairro Ponta Grossa para estas espécies pertencem à categoria de uso místico.

Plantas com um baixo Valor de Uso podem apresentar um alto índice de concordância entre suas indicações de uso, como ocorre com Lippia alba, Mikania laevigata. Plectranthus neochilus, Punica granatum, Sechium edule e Sphagneticola trilobata. Aloe arborescens (babosa) possui o maior índice de Valor de Uso, mas somente apresentou um percentual de 26 para CUPc. Aloe arborescens foi a espécie mais citada por diferentes informantes, sendo mencionados 37 usos diferentes. Porém, a concordância entre os informantes sobre a utilização dela foi relativamente baixa, sendo o uso principal para curar "feridas".

Vinte e sete espécies foram citadas em, no mínimo, seis das oito regiões estabelecidas para o bairro. Achyrocline satureioides, Aloe arborescens, Aloysia citrodora, Citrus $\times$ aurantium, Citrus limon, Cunila microcephala, Cuphea carthagenensis, Cymbopogon citratus, Eugenia uniflora, Foeniculum vulgare, Lippia alba, Plectranthus barbatus e Psidium guajava foram mencionadas em todas as regiões. Entre elas, somente Cuphea carthagenensis não está incluída nas 19 espécies com maior CUPc e somente Lippia alba está excluída das 21 espécies com maior Valor de Uso para a comunidade.

As espécies Persea americana, Phyllanthus tenellus, Punica granatum, Rosmarinus officinalis, Tanacetum vulgare e Zea mays foram mencionadas em sete das oito regiões do bairro. Phyllanthus tenellus, Punica granatum e Zea mays não estão enquadradas entre as espécies mais importantes para a população e somente Tanacetum vulgare e Punica granatum contêm o valor de CUPc maior que $24 \%$. Exceto estas, as outras espécies apresentam usos variados, porém diferentes entre si. As espécies citadas em seis das oito regiões foram Aloysia gratissima, Artemisia absinthium, Citrus reticulata, Ocimum basilicum, Plectranthus neochilus, Sechium edule, Sphagneticola trilobata e Tradescantia zebrina. Entre estas, somente Artemisia absinthium e Ocimum basilicum são consideradas com maior importância para a comunidade. Artemisia absinthium e Plectranthus neochilus possuem CUPc maior que $24 \%$. Os usos mencionados para as demais espécies não são coerentes entre si.

O índice de Valor de Uso para as espécies e famílias mostra o quão importante cada uma delas é para a comunidade estudada. Como $48 \%$ das 21 espécies mais importantes são nativas nas América, sendo 38\% delas nativas no Rio Grande do Sul, este índice pode ser um relevante argumento para a promoção da sua conservação em seu ambiente natural. Um aspecto importante para a conservação é estimular o cultivo das plantas utilizadas pelas populações, através de hortos comunitários ou em quintais ou pátios, como observado na comunidade estudada. Para estimular o cultivo, é necessário que haja investimentos em técnicas, principalmente para as espécies nativas. $\mathrm{O}$ cultivo reduz a retirada das plantas de seu ambiente natural e ainda, pode ser utilizado como fonte de renda para as comunidades e/ ou pequenos produtores (Ming 1994).

Este trabalho pode servir de referência para futuros estudos farmacológicos com espécies vegetais, por indicar algumas espécies com um potencial promissor para alívio ou cura dos sintomas ou doenças citados. Através do CUPc são apontados os usos mais difundidos e aceitos pela população para uma espécie. A aceitação de um uso por uma população implica em maior segurança quanto à relativa eficácia do uso proposto, pois ele é provavelmente utilizado ou conhecido por muitos informantes. Além disto, a difusão destes usos dentro de uma comunidade não parece ser recente, indicando que a espécie já é utilizada para determinados sintomas ou doenças há algum tempo.

\section{Agradecimentos}

Aos funcionários do Posto de Saúde da Família de Ponta Grossa, e aos moradores do bairro Ponta 
Grossa, Porto Alegre, Rio Grande do Sul; aos taxonomistas Cristiane Gonçalves, Daiane Bauer, Daniel Ruchel, Mara Rejane Ritter, Marcos Sobral, Nelson Ivo Matzenbacher, Rafael Trevisan, Renato Aquino Záchia, Rosana Maria Senna, Rose Bortoluzzi, Sérgio Augusto de Loretto Bordignon e Sonia Hefler, pela identificação de algumas espécies; aos funcionários do herbário ICN pela ajuda prestada.

\section{Referências bibliográficas}

Alexiades, M.N. 1996. Collecting ethnobotanical data: an introduction to basic concepts and techniques. Pp. 53-94. In: M.N. Alexiades (ed.). Guidelines for ethnobotanical field collectors. New York, The New York Botanical Garden.

Amorozo, M.C. \& Gély, A. 1988. Uso das plantas medicinais por caboclos do baixo Amazonas. Barcarena, PA, Brasil. Boletim do Museu Paranaense "Emílio Goeldi", Nova Série, Botânica 4(1): 47-131.
Friedman, J.; Yaniv, Z.; Dafni, A. \& Palewitch, D. 1986. A preliminary classification of the healing potential of medicinal plants, based on a rational analysis of an ethnopharmacological field survey among Bedouins in the Negev Desert, Israel. Journal of Ethnopharmacology 16: 275-287.

Ming, L.C. 1994. Estudo e pesquisa de plantas medicinais na agronomia. Horticultura Brasileira 12(1): 3-9.

Phillips, O. \& Gentry, A.H. 1993. The useful plants of Tambopata, Peru: I. Statistical hypotheses tests with a new quantitative technique. Economic Botany 47(1): 15-32.

Phillips, O.L. 1996. Some quantitative methods for analyzing ethnobotanical knowledge. Pp. 171-198. In: M.N. Alexiades (ed.). Selected guidelines for ethnobotanical research: a field manual. New York, The New York Botanical Garden.

Vendruscolo, G.S.; Rates, S.M.K. \& Mentz, L.A. 2005. Dados $\mathrm{qu}$ [imicos e farmacológicos sobre as plantas utilizadas como medicinais pela comunidade do bairro Ponta Grossa, Porto Alegre, Rio Grande do Sul. Revista Brasileira de Farmacognosia 15(4): 361-372. 